\title{
ArcheoSciences
}

Revue d'archéométrie

\section{Géochimie isotopique du plomb en archéologie minière et métallurgique}

Exemple du Mont Lozère dans les Cévennes

Lead isotopes in mining archaeology and paleometallurgy: An example from

Mont Lozère Massif (Cévennes, France)

\section{Sandrine Baron, Cécile Mahé Le Carlier et Alain Ploquin}

\section{OpenEdition}

Journals

Édition électronique

URL : https://journals.openedition.org/archeosciences/2723

DOI : $10.4000 /$ archeosciences. 2723

ISSN : 2104-3728

Éditeur

Presses universitaires de Rennes

Édition imprimée

Date de publication : 10 avril 2010

Pagination : 149-147

ISBN : 978-2-7535-1407-2

ISSN : $1960-1360$

\section{Référence électronique}

Sandrine Baron, Cécile Mahé Le Carlier et Alain Ploquin, « Géochimie isotopique du plomb en archéologie minière et métallurgique », ArcheoSciences [En ligne], 34 | 2010, mis en ligne le 11 avril 2013, consulté le 01 février 2022. URL : http://journals.openedition.org/archeosciences/2723 ; DOI : https://doi.org/10.4000/archeosciences.2723 


\title{
Géochimie isotopique du plomb en archéologie minière et métallurgique : exemple du Mont Lozère dans les Cévennes
}

\author{
Lead isotopes in mining archaeology and paleometallurgy: \\ An example from Mont Lozère Massif(Cévennes, France)
}

Sandrine Baron*, Cécile Mahé-Le Carlier ${ }^{* *}$ et Alain Ploquin***

\begin{abstract}
Résumé : La géochimie isotopique du plomb est un outil utilisé dans le domaine de l'archéologie minière et métallurgique. Dans le cadre d'une approche interdisciplinaire i.e. par l'implication active d'archéologues, historiens, géographes, palynologues et géologues, elle permet de (i) restituer la filiation mine - objet et l'histoire des activités minières et métallurgiques dans le paléo environnement et (ii) de quantifier et caractériser l'héritage minier dans l'environnement actuel. Ces études ont été menées sur le Mont-Lozère (Cévennes, France), dans le cadre du PCR « Plomb Ancien du Mont-Lozère " qui est un Projet Collectif de Recherche financé par le Ministère de la Culture.
\end{abstract}

Abstract : The geochemistry of lead isotopes is a tool basically used in mining and metallurgical archaeology. Combining with an active interdisciplinary approach i.e. archaeologists, historians, geographers, palynologists, geologists... it allows i) the restitution of mine - metal filiations and the restitution of mining and metallurgical activities in the paleo environment and (ii) the quantification and characterisation of mining heritage in the actual environment. Our study carried out on the Mont-Lozère massif (Cévennes, France), inside a project intituled "Plomb ancien du Mont-Lozère" (financed by the Ministry of (Culture), constitutes a good example of interdisciplinary research.

Mots clé : géochimie, archéologie, isotopes du plomb, approche interdisciplinaire.

Key words : geochemistry, archaeology, lead isotopes, interdisciplinary approach.

\section{LES ISOTOPES DU PLOMB : UN OUTIL DE CARACTÉRISATION DE L'HÉRITAGE MINIER}

Pour résoudre les problématiques archéologiques liées aux matières premières métalliques et aux objets métalliques, le domaine de l'archéométrie met en œuvre des techniques analytiques et des modes de raisonnement issus d'autres disciplines, notamment des Sciences de la Terre et des Matériaux, et plus récemment de l'Environnement. Dans le domaine particulier traité ici, la géochimie (i.e. chimie appli-

\footnotetext{
* Laboratoire des Travaux de Recherches Archéologiques sur les Cultures, les Espaces et les Sociétés, Université Toulouse 2 Le Mirail, CNRS UMR 5608 - Maison de la Recherche, 5 allée Antonio-Machado, 31058 Toulouse Cedex 09, France. (sbaron@univ-tlse2.fr)

** Centre de Recherches en Archéologie, Archéosciences, Histoire, Université Rennes 1, CNRS UMR 6566, Bat. 24-25-Campus de Beaulieu, 35042 Rennes, Cedex, France.

*** 8, Chemin de l'hermitage, 54850, Messein, France.
} 
quée à la géologie) apparaît comme un outil bien adapté, à condition de bien poser et peser les contraintes à la fois en géochimie et en archéologie.

\section{Principe général des isotopes du plomb}

Le plomb $(\mathrm{Pb})$ constitue un système isotopique abondamment utilisé (Faure, 1986). Ses champs d'applications sont larges : géochronologie, cosmochimie, traçage de l'origine et de l'évolution des minéralisations, problématiques environnementales et plus récemment l'archéométrie.

Malgré les récents développements en géochimie isotopique sur de nouveaux traceurs de sources comme le cuivre et le zinc par exemple (Klein et al., 2004), le plomb reste cependant unique de par sa variabilité isotopique sur Terre mais aussi de par les comportements respectifs différents de ses isotopes $\left({ }^{208} \mathrm{~Pb},{ }^{207} \mathrm{~Pb},{ }^{206} \mathrm{~Pb}\right.$ et $\left.{ }^{204} \mathrm{~Pb}\right)$. Les isotopes $\mathrm{du} \mathrm{Pb}$ sont donc d'excellents traceurs de sources.

Le plomb possède 3 isotopes radiogéniques (qui provient de la décroissance radioactive d'un isotope dit "père ") que l'on peut considérer comme "stables " à échelle de l'âge de la Terre : ${ }^{206} \mathrm{~Pb},{ }^{207} \mathrm{~Pb}$ et ${ }^{208} \mathrm{~Pb}$. Ces 3 isotopes sont produits de façon continue au cours du temps via la chaîne de désintégration d'isotopes radioactifs à partir de l'uranium (U) et du thorium (Th) : ${ }^{238} \mathrm{U},{ }^{235} \mathrm{U}$ et ${ }^{232} \mathrm{Th}$ respectivement. Le quatrième isotope, le ${ }^{204} \mathrm{~Pb}$, n'est pas radiogénique : il ne résulte pas de la décroissance radioactive d'un isotope "père ", son abondance dans les réservoirs (atmosphère, croûte continentale, minéralisations...) est restée la même depuis la formation de la Terre.

Les comportements géochimiques du Pb, de l'U et du Th sont différents. Chaque réservoir possède des rapports initiaux $\mathrm{U} / \mathrm{Pb}$ et $\mathrm{Th} / \mathrm{Pb}$ qui ont évolué différemment au cours du temps et des évènements géologiques. Ainsi, chaque réservoir possède ses propres compositions isotopiques pour un âge donné. Par conséquent, différents dépôts minéralisés peuvent posséder leur propre signature isotopique du plomb qui est dépendante de son origine géologique.

\section{Implications en archéologie minière}

Les utilisations de ce système isotopique en archéologie minière et métallurgique sont les suivantes :

La restitution des fliations des métaux. L'objectif est de faire le lien le long de la chaîne opératoire entre : a) les districts miniers (parfois bien attestés ou non archéologiquement), pour une région donnée, $b$ ) les ateliers de production du métal, généralement attestés par la présence de déchets métallurgiques (i.e. scories), et $c$ ) les lingots et/ou objets retrouvés souvent hors contexte minier ou sites métal- lurgiques et dans des zones géographiques plus ou moins éloignées. La similitude (ou non) entre compositions isotopiques de plomb de districts miniers, de scories et d'objets métalliques divers permet d'argumenter sur la provenance des matières premières ayant servies à l'élaboration d'objets divers anciens. Ces relations apportent de nombreuses informations quant aux échanges économiques des anciennes civilisations.

La restitution historique des activités minières et métallurgiques vue au travers du paléo environnement. Pour une région donnée, l'histoire des activités anthropiques anciennes peut être restituée par l'étude temporelle d'archives environnementales telles que les carottes de tourbe, de sédiment et de glace. En effet, les anciens centres d'exploitation de minerais et/ou de productions de métaux ont engendré des poussières chargées en métaux lourds divers et en poussières de roches encaissantes. Ces dernières retombent, entre autres, dans les tourbières à proximité soit par voie atmosphérique soit par voie terrestre (via les eaux de ruissellement transportant des sédiments riches en métaux lourds). La lecture de ces carottes permet de restituer l'historique des dépôts liés aux activités métallurgiques anciennes mais aussi de dépister des activités méconnues. Implicitement, cela permet d'orienter les recherches archéologiques.

La quantification des polluants dans l'environnement actuel. L'environnement actuel étant le réceptacle des activités passées, la quantification des métaux lourds puis leur caractérisation isotopique permet d'estimer la contribution des activités passées dans le budget total des pollutions actuelles. Un paysage, qualifié a priori de "naturel ", peut être un lieu où d'anciennes activités métallurgiques importantes ont pris place par le passé et ce, bien avant la Révolution Industrielle.

\section{UN CAS D'ESPÈCE : LES ACTIVITÉS MÉTALLUR- GIQUES MÉDIÉVALES DU MONT LOZĖRE}

\section{Le massif du Mont Lozère : lieu d'importantes activités métallurgiques}

Quatre-vingts sites à scories ont jusqu'ici été inventoriés sur le massif du Mont-Lozère, au cœur du Parc National des Cévennes. Au pourtour du Mont-Lozère, de nombreuses mines (ou indices) de minerais de plomb plus ou moins riche en argent (sous forme de galènes argentifères) sont répertoriées (Ploquin et al., 2003). Les différents secteurs miniers présents sur le pourtour du massif granitique sont connus, à travers des anciens textes de l'école des Mines 
d'Alès ou des archives du BRGM (Bureau de Recherches Géologiques et Minières), pour avoir été exploité « par les anciens " mais ces mines n’ont pas été étudiées ni fouillées (Laurent, 2005). Les datations ${ }^{14} \mathrm{C}$ réalisées sur des charbons en relation stratigraphique avec des scories convergent vers une période médiévale début $\mathrm{XI}^{\mathrm{e}}$-XIV ${ }^{\mathrm{e}}$ siècle voire début $\mathrm{XV}^{\mathrm{e}}$ siècle. Ces activités métallurgiques ont engendré une pollution dont les conséquences sont visibles de nos jours. En effet, beaucoup de sites se traduisent soit par des zones dénudées où aucune végétation ne se développe (à part certaines plantes métallophiles ou indifférentes et opportunistes) ou bien par des zones constituées d'une végétation perturbée qui se caractérise par de rares pins et bouleaux rabougris pratiquement sans feuille et/ou précocement morts. Les zones polluées peuvent s'étaler au sol sur une distance de 300 mètres (riches ou non en scories), les eaux de ruissellements se chargeant de répandre les métaux lourds dans l'environnement immédiat (fig. 1).

La prospection en amont d'épandages de scories, mais également des arrachements ponctuels ou des taupinières ont permis de déceler des sites enfouis par des colluvions. Ainsi, sous à peine $30 \mathrm{~cm}$ de profondeur, il a été parfois possible de retrouver les sols à vestiges d'ateliers médiévaux. Au-delà de ces effets visibles, les différents réservoirs environnementaux (sols, végétaux, atmosphère, tourbières...) stockent ces métaux lourds à échelle régionale puis à terme, à échelle globale (Nriagu and Pacyna, 1988).

\section{Validation de la traçabilité minerais-ateliers- environnement}

Dans un premier temps, il est essentiel de valider l'utilisation du traceur choisit le long de la chaîne opératoire de production du plomb (fig. 2). Il s'agit d'estimer l'impact de la phase "réduction métallurgique " sur les compositions isotopiques de plomb des différents produits métallurgiques.

\section{Les expérimentations archéologiques: validité du traceur et compréhension du procédé métallurgique mis en æuvre}

Des études antérieures (avec les précisions analytiques acceptables) ont émis l'hypothèse que la réduction du minerai pouvait perturber les signatures isotopiques du plomb. Les fumées, produisant des pertes de masse importantes, sont susceptibles de changer la composition du traceur entre les minerais et les scories à l'échelle du four de réduction (Budd et al., 1995).

Afin de s'autoriser à établir des comparaisons entre les minerais et les scories en vue de la restitution de la filiation, il a fallu s'assurer que les compositions isotopiques $\mathrm{du} \mathrm{Pb}$ restaient identiques pendant les opérations métallurgiques.

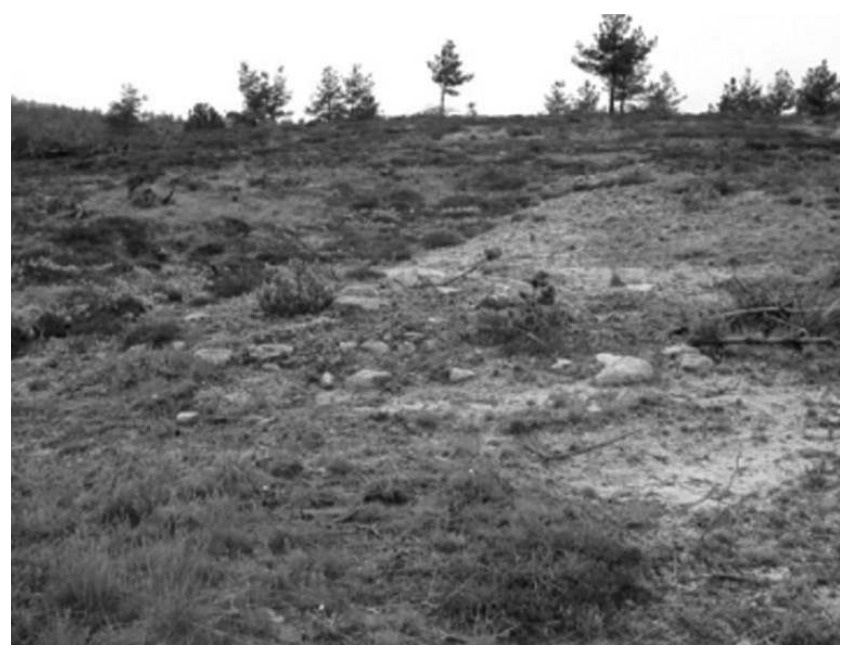

Figure 1 : Exemple d'un site pollué sur le Mont-Lozère. La partie polluée se caractérise par des zones d'épandage de scories (coin droit, en bas de la photographie). La partie non polluée se situe dans le coin gauche de la photographie, en haut de la colline. Photo A. Ploquin. Figure 1: Example of a polluted site on Mont-Lozère. The polluted area is characterized by zones of slag spreading (right corner, at the bottom of the photography). The not polluted area is located on the left corner of the photography, at the top of the hill. Photo: A.Ploquin.

Ainsi, des reconstitutions archéologiques expérimentales de " réduction " de la galène ont été effectuées sur la plateforme expérimentale de Melle, dans le département des Deux-Sèvres (dirigée par Florian Téreygeol). Elles sont de deux types et menées dans un même type de four à cuve :

- soit une "réduction » du minerai sans ajout;

- soit avec un ajout de plomb métallique en début d'opération. Cet emplombage est mentionné par Biringuccio (1540). Ce procédé permettrait de favoriser la récupération de l'argent directement à partir de la galène. Nos scories du Mont-Lozère sont issues de l'élaboration de plomb d'œuvre (riche en argent) à partir de galènes argentifères, d'où le choix de tester ce procédé.

Tous les produits de chaque expérimentation ont été échantillonnés (y compris les fumées à divers stades de l'opération) afin d'estimer les bilans isotopiques. Pour de plus amples informations sur la conduite des expérimentations et les discussions, se reporter dans Baron (2005) et Baron et al. (2009).

Ces expérimentations archéologiques ont montré que des variations isotopiques $\mathrm{du} \mathrm{Pb}$, significatives, sont mesurables au sein d'un même four métallurgique. Elles seraient probablement dues à l'hétérogénéité des minerais sources mais aussi à l'hétérogénéité des conditions physico-chimiques à l'intérieur du four. Ainsi, il est nécessaire de mesurer plusieurs scories au sein d'un même atelier de réduction. 


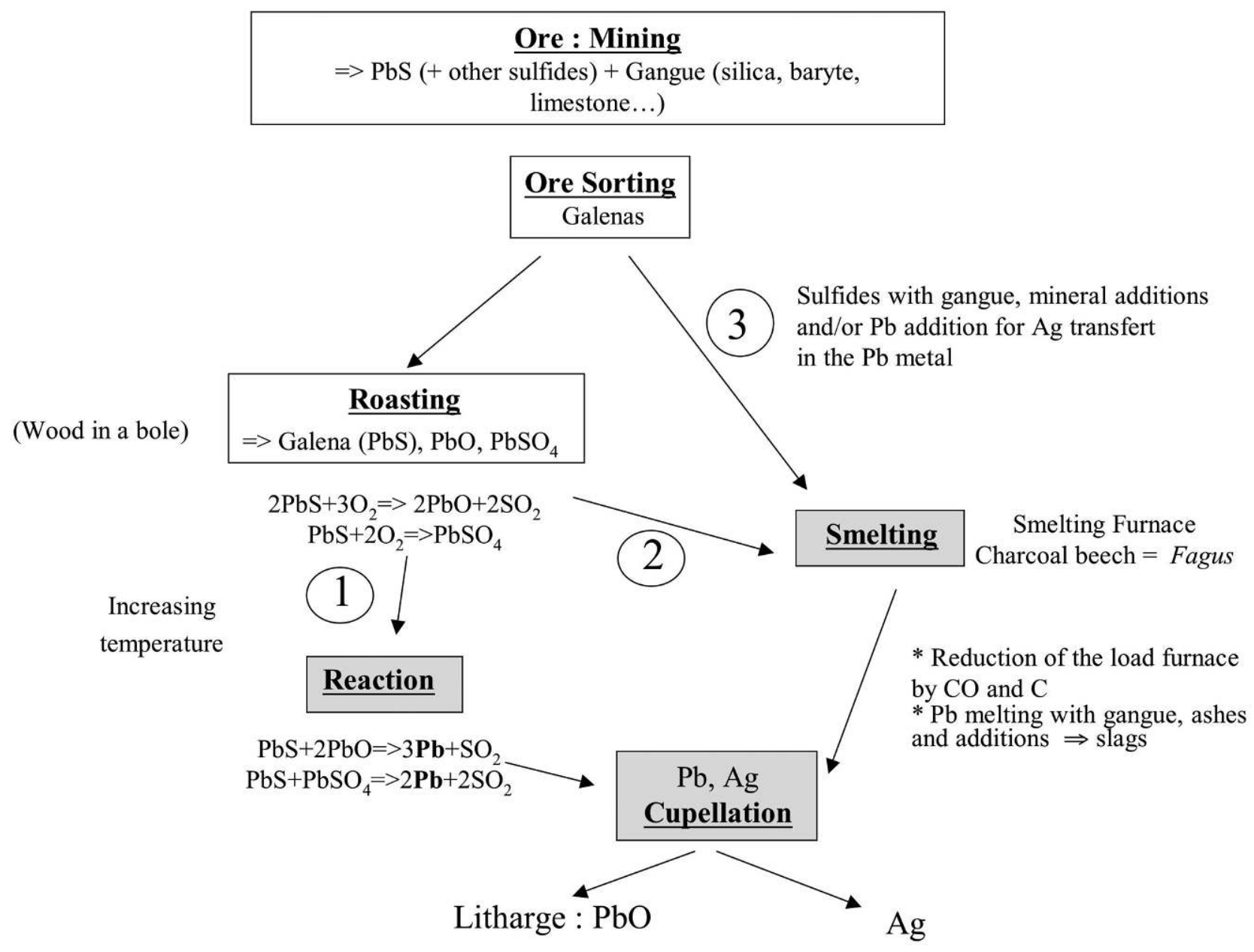

Figure 2: Schéma de la chaîne opératoire ancienne du plomb argentifère (extrait des travaux de Baron et al., 2009, figure 1). La phase " coupellation " n’a pas été décelée dans les ateliers du Mont-Lozère.

Figure 2: The ancient chaine operatoire of lead argentiferous (extract from Baron et al., 2009, figure 1). The cupellation step was not realised on the Mont-Lozère workshops.

Les scories donnent la moyenne des compositions chimiques et isotopiques de $\mathrm{Pb}$ des minerais. Elles sont géographiquement en place et souvent pas très loin des aires d'extractions de minerais, ce qui permet d'affiner la traçabilité dans le cas de minerais hétérogènes. De plus, dans le cas où les minerais ne sont pas attestés par l'archéologie minière comme ayant été exploités par les Anciens, il est plus prudent d'utiliser les scories (en contexte archéologique bien sûr) pour restituer la filiation des métaux plutôt que les minerais eux-mêmes. Par contre, dans le cas contraire, où les scories sont rares (cas de la métallurgie de l'or par exemple), le minerai est le seul candidat pertinent pour restituer la filiation, encore faut-il s'assurer que ce dernier a bien été exploité et qui plus est, aux périodes historiques correspondant à la problématique.
Malgré la haute résolution de nos analyses, l'ajout de $\mathrm{Pb}$ n'a pas de contribution significative dans les compositions isotopiques de $\mathrm{Pb}$ des fumées, sauf en tout début d'opération, validant une fois de plus, la pertinence de notre traceur le long de la chaîne opératoire. Dans notre reconstitution, nous avons sciemment utilisé un plomb nettement étranger (i.e. dont la composition isotopique est très différente). L'utilisation éventuelle, dans les ateliers de réduction, d'un plomb indigène (ce qui semble assez probable) serait donc difficile à déceler.

Les études métallographiques ont permis de démontrer que l'ajout de plomb en début de réduction permettait de favoriser la réduction de la galène argentifère et donc, d'optimiser la récupération de l'argent. 
Ces expériences ont également montré que les compositions isotopiques des produits de réduction sont celles $\mathrm{du}$ minerai de départ et cela nous autorisent à établir une filiation entre les fumées, les déchets métallurgiques, les sources du minerai et les traces dans l'environnement, dans le cas du Mont-Lozère (Baron et al., 2009).

\section{L'origine des minerais pour la production de plomb argentifère sur le Mont Lozère}

Aucune information historique sur l'exploitation des mines dans cette région n'est disponible pour les époques médiévales (Laurent, 2005). Il s'agissait de retrouver les secteurs miniers ayant alimenté les ateliers métallurgiques médiévaux.

Dans l'encaissant du massif granitique du Mont-Lozère, différents secteurs miniers sont susceptibles d'avoir alimenté les activités métallurgiques; leurs haldes (déchets rocheux de l'extraction du minerai) ont été échantillonnées afin d'attribuer une signature isotopique de $\mathrm{Pb}$ de chaque district minier présent au pourtour du massif.

Les principaux produits disponibles sur les sites de production sont les scories et leurs billes de plomb (incluses dans les matrices silicatées des scories). Les sites médiévaux ont été caractérisés pour leur composition isotopique de $\mathrm{Pb}$. Plusieurs sites d'ateliers médiévaux ont été mesurés afin d'estimer la dispersion (ou non) des signatures isotopiques et d'autre part plusieurs scories ont été échantillonnées sur un même site afin d'estimer si l'approvisionnement en minerai, par atelier, est homogène ou non.

Les signatures isotopiques $\mathrm{du} \mathrm{Pb}$ des sites métallurgiques médiévaux ont été comparées à celles des minéralisations présentent au pourtour du massif (Baron et al., 2006a).

Il a été ainsi démontré que les sites d'ateliers métallurgiques ont été approvisionnés par des minerais localisés au sud-ouest du massif du Mont-Lozère (fig. 3). Ce résultat est assez surprenant car la majorité des sites d'ateliers découverts (75\%) sont localisés sur le versant nord du massif, où des mines de plomb argentifères sont également inventoriées et situées à proximité des sites à scories (fig. 3). Les futures recherches archéologiques porteront sur les secteurs miniers retenus par les résultats des isotopes du plomb, par exemple, le filon du Montet, dans le secteur Montmirat dont la morphologie des indices d'exploitations en "pingen " évoque fortement une exploitation médiévale.

Par conséquent, les hypothèses les plus probables pour expliquer la localisation géographique de ces sites à scories sur le massif sont doubles :

- d'une part, elles seraient liées aux droits d'exploitation et d'usage des mines à l'époque médiévale (Bailly-Maitre 2002);
- d'autres part, elles seraient liées également à des causes paléo environnementales dues à la localisation de la hêtraie à cette époque obligeant les anciens à déplacer le minerai à proximité du combustible (Allée, 2003; Allée et al., ce volume).

\section{Reconstitution historique de la pollution médiévale dans l'environnement ancien}

Les valeurs isotopiques de $\mathrm{Pb}$ des scories ont été utilisées comme traceur des activités anthropiques médiévales dans l'environnement.

Létude des paléo pollutions, à des échelles régionales, a débuté en France depuis très peu de temps (Monna et al. 2004a, b; Baron et al., 2005; Arnaud et al., 2006; JouffroyBapicot et al., 2006).

Les analyses chimiques élémentaires et isotopiques qui ont été menées sur une carotte de tourbe des Narses Mortes, en collaboration avec les palynologues, les archéologues, les géographes et les historiens, nous ont permis de comprendre l'histoire des activités anthropiques, à l'échelle du massif, depuis près de 2500 ans (Baron et al., 2005; Pulido, 2006). La figure 4 présente le diagramme palynologique simplifié et le rapport des concentrations en plomb sur celles de l'aluminium. Ce rapport est en fait une normalisation qui permet de mettre en évidence l'excès de métaux lourds par rapport au plomb naturel local, pour un niveau de tourbe donné.

Dans les niveaux inférieurs de la carotte de tourbe, le rapport $\mathrm{Pb} / \mathrm{Al}$ est très faible. Par conséquent, aucune activité métallurgique n'a lieu sur le massif : aucun excès de plomb n'est enregistré dans la carotte.

Par contre, à $118 \mathrm{~cm}$ de profondeur, au second âge du Fer, l'étude du profil révèle l'existence d'une activité métallurgique débutant à environ $250 \mathrm{~B}$. C. Cette activité se traduit par une augmentation très nette et très forte du rapport $\mathrm{Pb} / \mathrm{Al}$ indiquant l'introduction d'excès important de plomb dans les niveaux tourbeux, bien supérieur aux apports de plomb naturel. Cette activité métallurgique semble durer jusqu'à environ 50 B.C.; elle serait donc gauloise et pourrait être liée à « l'argent des Gabales » cité par Strabon et César.

Puis le signal $\mathrm{Pb} / \mathrm{Al}$ diminue traduisant qu'aucune activité métallurgique se ne produit sur le massif.

Aux environs de $50 \mathrm{~cm}$ de profondeur, un nouveau pic du rapport $\mathrm{Pb} / \mathrm{Al}$ débute. Il s'agit de l'activité métallurgique médiévale. Les compositions isotopiques de $\mathrm{Pb}$ associées à ces mêmes niveaux sont celles des scories qui sont présentes sur le massif. Cependant, les rapports isotopiques $\mathrm{du} \mathrm{Pb}$ dû à la Révolution Industrielle masquent la pollution médiévale dans les couches plus jeunes ( $-30 \mathrm{~cm}$ de profondeur), empêchant d'estimer la durée des activités minières locales. 


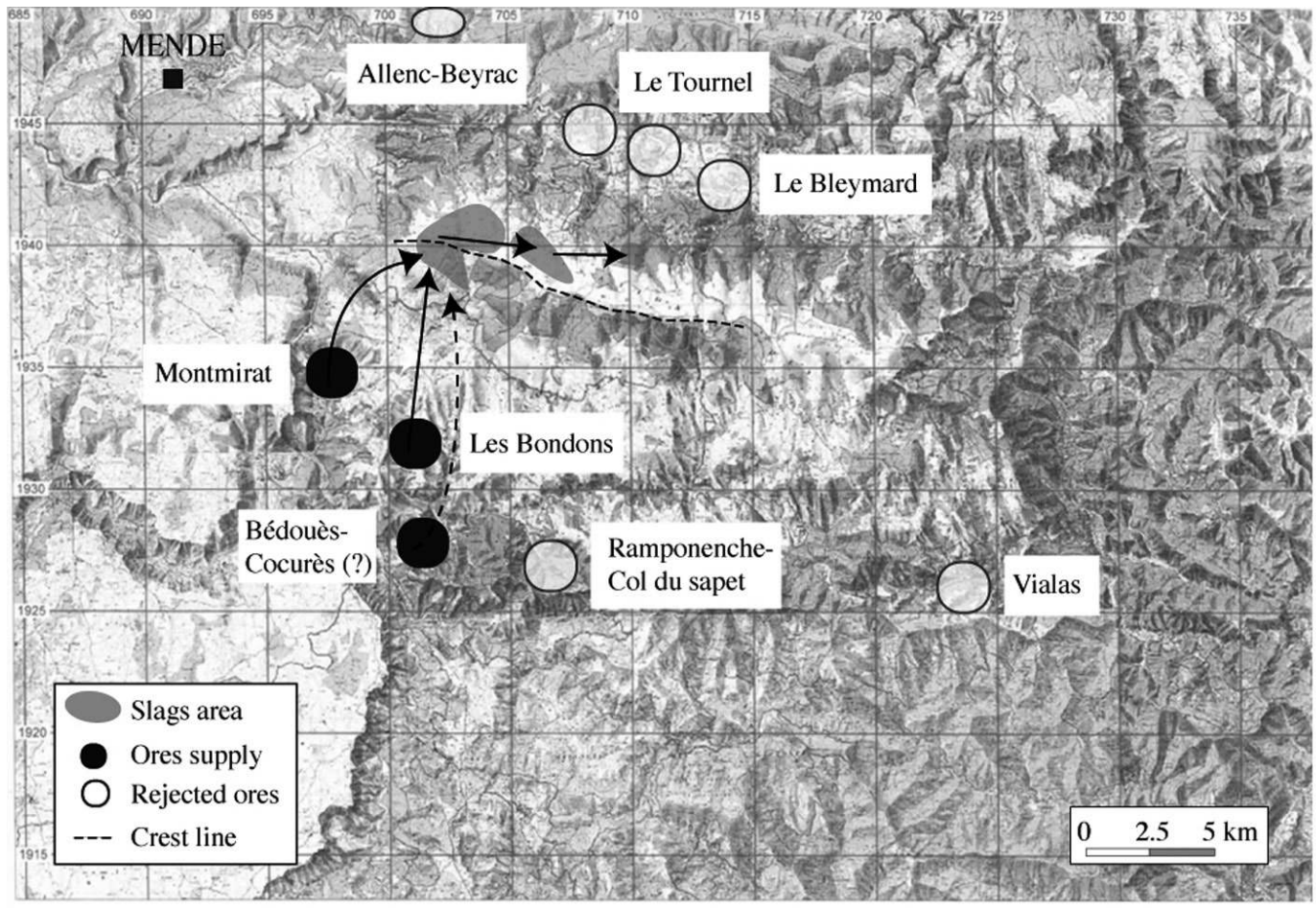

Figure 3 : Restitution de la filiation minerais-scories sur le Mont-Lozère (extrait des travaux de Baron et al., 2006a, figure 5).

Figure 3: Restitution of the ores - slag filiation on Mont-Lozère (extract from Baron et al., 2006a, figure 5).

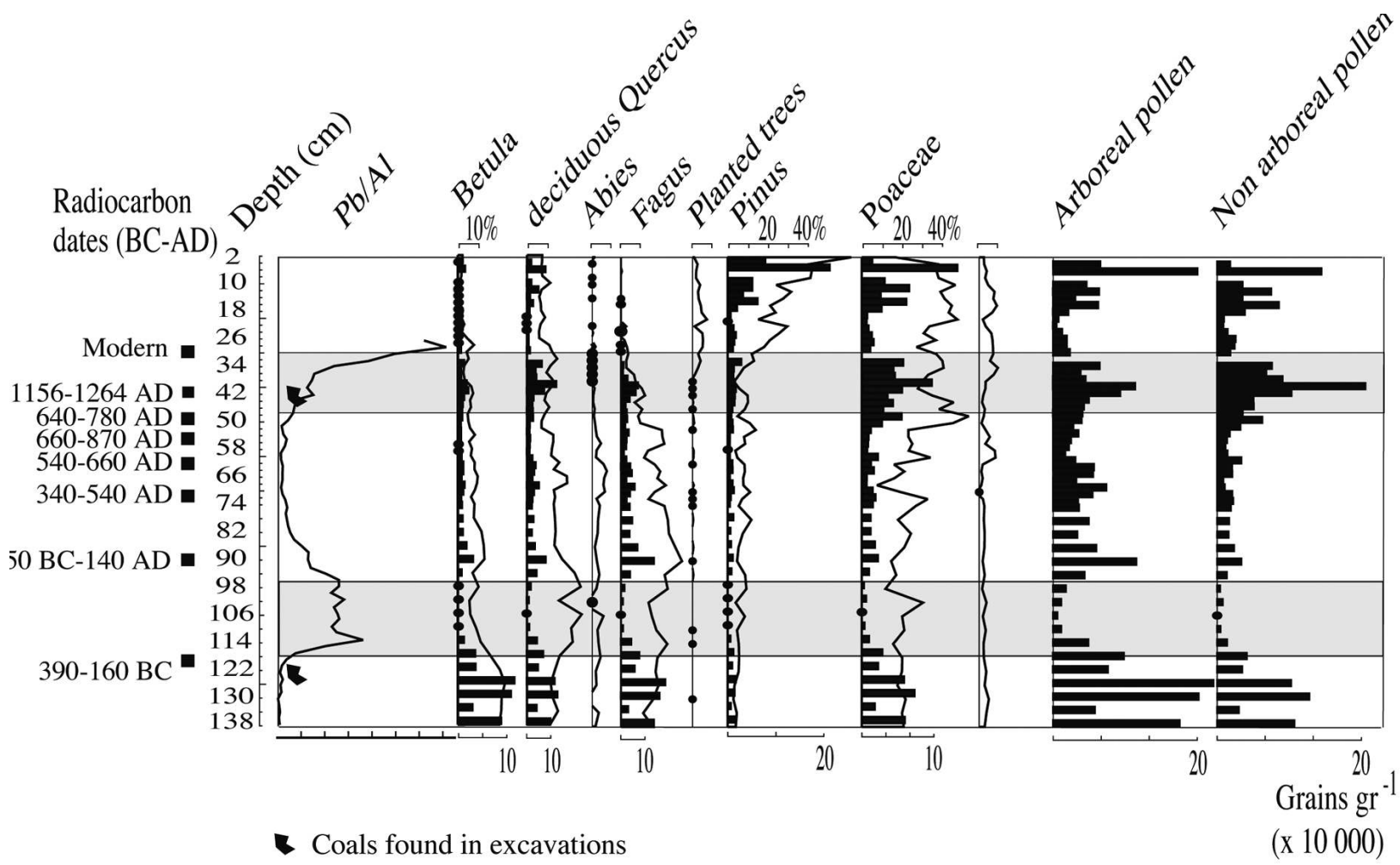

Figure 4 : Diagramme palynologique couplé avec les concentrations en plomb normalisées a l'aluminium de la carotte de tourbe des Narses Mortes (extrait des travaux de Baron et al., 2005, figure 4). La bande grise supérieure correspond à la métallurgie médiévale et la bande inférieure à la métallurgie gauloise.

Figure 4: Palynological diagram combined with lead concentrations normalised to aluminium content of Narses Mortes peat core (extract from Baron et al., 2005, figure 4). The superior grey band corresponds to the medieval metallurgy and the lower grey band to celtic metallurgy. 
Les compositions isotopiques du plomb des activités métallurgiques gauloises sont similaires à celles des activités métallurgiques médiévales. Cela laisse supposer que les mêmes gisements auraient été exploités à deux périodes différentes.

\section{Impact persistant dans l'environnement récent}

\section{La dispersion de la pollution médiévale dans les sols}

Les analyses élémentaires de vestiges de sols d'ateliers présentent des teneurs en métaux lourds très élevées (jusqu'à $25 \%$ en masse) comparativement au bruit de fond naturel local. Les sols d'ateliers possèdent les mêmes compositions isotopiques de $\mathrm{Pb}$ que les scories et $40 \%$ à $100 \%$ de leur plomb provient des activités métallurgiques médiévales. Les plus pollués sont les anciens sols d'occupations médiévaux i.e. les anciens sols d'ateliers où le métal était élaboré. Ces sols d'ateliers (aires de travail essentiellement et/ou parfois sols présents autour de four pour un des sites) sont composés de morceaux de minerais qui ont crépité hors du four, de poussières, fumées, morceaux de charbons, petits fragments de scories épandus lors de l'ouverture du four.

La plupart des métaux lourds comme le plomb, l'antimoine $(\mathrm{Sb})$, l'arsenic (As) et le cuivre $(\mathrm{Cu})$ dérivent de la même source de pollution et présentent le même type de dispersion dans les sols. Le zinc $(\mathrm{Zn})$ est en déficit relatif par rapport à $\mathrm{Pb}, \mathrm{Cu}$, As et $\mathrm{Sb}$. Les sols semblent « lessivés " d'une partie de leur $\mathrm{Zn}$, suggérant que ce dernier est sujet à des processus de remobilisation différents des autres métaux dans cette étude, dont une absorption préférentielle par des végétaux.

Bien qu'ayant un comportement semblable, $\mathrm{Cu}$ est cependant en excès dans tous les sols; cet excès est vraisemblablement dû à un dernier tri du minerai sur place afin d'y éliminer la chalcopyrite, liée au minerai, avant son chargement dans les fours. En effet, une trop grande quantité de chalcopyrite induirait la formation de mattes (sulfure de fer et de cuivre) qui est une source de problèmes pour le fonctionnement du four et entraîne des pertes en argent.

De plus, une première étude sur l'altération des scories (non publiée) a montré que le principal pourvoyeur de la pollution n'était pas la lixiviation des scories dans l'environnement mais la dislocation (i.e. dispersion physique) des anciens sols d'ateliers médiévaux avec le temps. Les teneurs en métaux lourds sont du même ordre de grandeur que celles de sols de sites industriels modernes (Baron et al., 2006b).

\section{Traces actuelles de la pollution atmosphérique médiévale}

Afin d'estimer la part du plomb médiéval dans la végétation actuelle, trois bouleaux ont été échantillonnés (racines, bois, tronc ou branchettes, écorces) : le bouleau 1 a été échantillonné sur l'épandage du site, le bouleau 2 juste en aval du site et le bouleau 3 à plus de 100 mètres à l'écart de la circulation des eaux et sédiments de ce même site. Il n'est présenté ici que les diagrammes des bouleaux 2 et 3 .

Les compositions isotopiques de $\mathrm{Pb}$ des bouleaux 2 et 3 (fig. 5) se placent entre deux pôles : celui de la scorie et celui de l'atmosphère (illustré par l'analyse isotopique de $\mathrm{Pb}$ des lichens épiphytes qui sont de bons indicateurs des retombées atmosphériques actuelles).

Les compositions isotopiques de $\mathrm{Pb}$ des échantillons de bois et des racines du bouleau 2 possèdent des compositions

Bouleau 2 : en aval du site

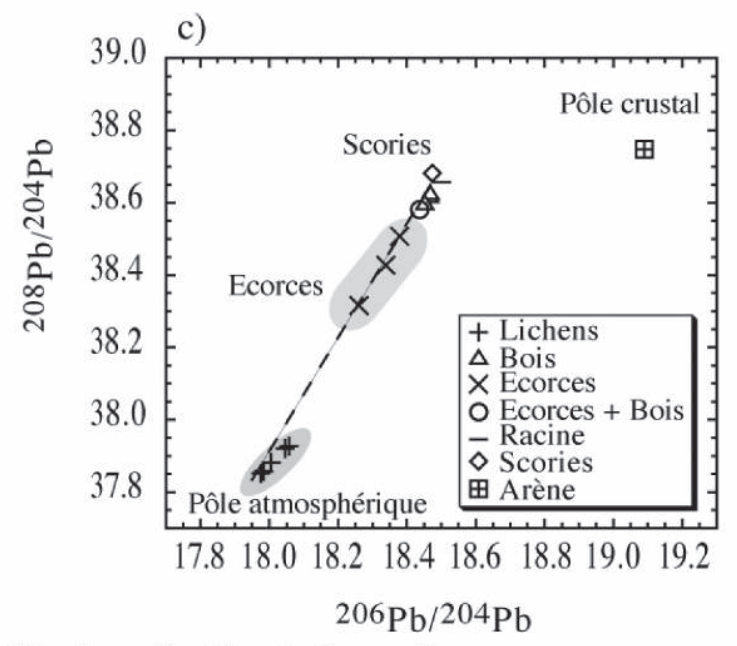

Bouleau 3 : témoin hors site

e)

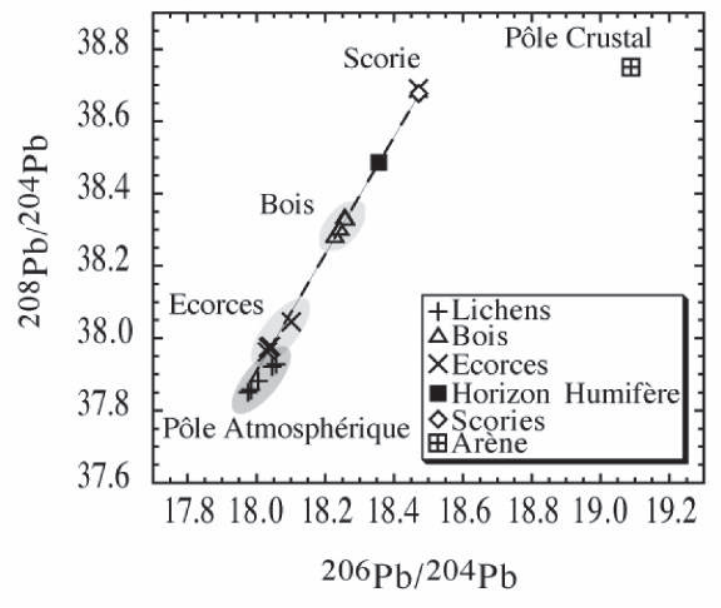

Figure 5 : Les compositions isotopiques de plomb ${ }^{208} \mathrm{~Pb} /{ }^{204} \mathrm{~Pb}$ en fonction de ${ }^{206} \mathrm{~Pb} /{ }^{204} \mathrm{~Pb}$ des bouleaux 2 et 3 échantillonnés sur le Mont-Lozère (extrait de la thèse de doctorat de Baron 2005, figure VI-12). L'arène granitique est celle du Mont-Lozère.

Figure 5: 208Pb/204Pb versus de 206Pb/204Pb diagram of birch 2 and 3 located on Mont-Lozère (extract from Ph. D thesis of Baron 2005, figure VI-12). The granitic sand comes from Mont-Lozère. 
similaires à celles de la scorie du site 8 (site à proximité duquel l'étude a été effectuée). Malgré l'éloignement de ce bouleau par rapport à l'atelier métallurgique (situé $150 \mathrm{~m}$ en amont), la contribution médiévale domine largement le budget $\mathrm{du} \mathrm{Pb}$ contenu dans les bois $(-98 \%)$. La quasi-totalité du $\mathrm{Pb}$ du bois de $\mathrm{B}-2$ provient actuellement du $\mathrm{Pb}$ des activités métallurgiques anciennes, stocké dans les sols et les déchets d'atelier, puis remobilisé ensuite par l'érosion et l'eau. Les écorces contiennent environ 80 à $65 \%$ de $\mathrm{Pb}$ provenant des activités métallurgiques et 20 à $35 \%$ de retombées atmosphériques modernes.

Les compositions isotopiques des bois du bouleau 3 (B-3), situés hors de l'épandage de pollution, permettent d'estimer que 70 à $80 \%$ du plomb est hérité de la métallurgie médiévale tandis que les écorces n'en contiennent plus que 5 à $15 \%$. L'hypothèse avancée pour expliquer cette pollution du bouleau 3 réside dans la composition même de ce sol. En effet, l'horizon humifère, qui est un "sol tourbeux " échantillonné à environ $30-40 \mathrm{~cm}$ de profondeur, possède une composition isotopique de $\mathrm{Pb}$ d'origine métallurgique. Cet horizon, probablement en surface à l'époque médiévale, devait recevoir les retombées de fumées issues de l'activité métallurgique. La tourbière a ensuite poursuivi sa croissance et ce niveau, actuellement enfoui, est aujourd'hui accessible par les racines du bouleau qui y puisent ses éléments. Par conséquent, via l'aubier, les polluants de la couche médiévale remontent à la surface et sont stockés dans le bois.

Il est important de noter que le plomb naturel («crustal») est à peine perceptible dans ce système environnemental et que seuls deux types de pollutions interviennent dans le budget total des teneurs en métaux lourds : la médiévale et la moderne (dans une moindre mesure pour cette dernière). Pour de plus amples informations sur les résultats et les modalités de calculs des contributions de $\mathrm{Pb}$ médiéval en pourcentage, se reporter ailleurs (Baron, 2005; Baron et al., 2007).

L'étude de ces bouleaux démontre que les métaux lourds issus de l'activité métallurgique médiévale sont remobilisés. Ainsi, les zones polluées ne se cantonnent pas essentiellement aux zones d'épandages en aval des sites; la pollution " ancienne " est probablement beaucoup plus étendue à l'échelle du massif.

\section{Conclusions}

L'utilisation de la géochimie (élémentaire et isotopique) dans le cadre d'une démarche interdisciplinaire (palynologie, géologie, histoire, archéologie, géographie...) a permis d'établir une démarche globale à l'échelle d'un territoire.
Cette étude a démontré que les sources de minerais utilisées pour les ateliers étaient des minéralisations situées au sud-ouest du massif. Ceci permet d'orienter les futures fouilles archéologiques sur le secteur retenu par les études isotopiques du plomb.

L'étude d'une carotte de tourbe a permis de restituer la chronologie de 2500 ans d'activité humaine sur le massif. Deux activités métallurgiques majeures ont été identifiées et caractérisées, la métallurgie médiévale et aussi le dépistage d'une métallurgie gauloise.

L'impact de ces activités métallurgiques reste visible sur le paysage actuel. En effet, les sols d'ateliers possèdent des teneurs en métaux lourds du même ordre de grandeur que des sols pollués actuels. Les études menées sur les bouleaux, soit sur site d'épandage à scories ou soit hors site d'épandage, montrent que la pollution médiévale est toujours très persistante dans le budget total des métaux lourds actuellement présent sur le massif et ce, dans différents supports environnementaux.

Notre héritage minier et métallurgique ante Révolution Industrielle est très mal connu de façon générale, et pourtant, leurs paléo pollutions présentent un impact environnemental encore visible de nos jours. Le cas du Mont Lozère est un bon exemple d'étude de cet héritage qui ne peut se concrétiser que par une approche interdisciplinaire.

\section{Remerciements}

Nous remercions le SRA (Services Régionaux de l'Archéologie) pour son soutien au PCR. La thèse de doctorat de Sandrine Baron a été financée par l'ADEME (Agence de l'Environnement et de la Maîtrise de l'Énergie) en co-financement avec la Région Languedoc-Roussillon. Nous remercions tout spécialement le maire de Saint-Étienne du Valdonnez (M. Claude Feybesse) pour son soutien logistique sur le terrain. Les analyses géochimiques ont été réalisées au laboratoire CRPG (Centre de Recherches Pétrographiques et Géochimiques, unité propre du CNRS à Nancy). Nous remercions aussi également le SARM (Service d'Analyses des Roches et des Minéraux, localisé au CRPG, à Nancy) et son directeur, Jean Carignan, pour son intérêt et son soutien.

\section{Bibliographie}

Allée, Ph., 2003. - Dynamiques hydro sédimentaires actuelles et holocènes dans les systèmes fluviaux de moyenne montagne (Limousin, Hautes Cévennes, Maures), Habilitation à diriger des Recherches, Université de Limoges, France. 
Arnaud, F., Serralongue, J., Winiarzki, T., Desmet et M., Paterne, M., 2006. - Pollution au plomb dans la Savoie Antique ( $\mathrm{II}^{\mathrm{e}}$-III ${ }^{\mathrm{e}}$ siècle apr. J.-C.) en relation avec une installation métallurgique de la cité de Vienne, Comptes-rendus, Géosciences, 338, 2006, p. 244-252.

BaIlly Maître, M.-Ch., 2002. - L'argent. Du minerai au pouvoir dans la France médiévale, éd. Picard, Paris, France.

BARON, S., 2005. - Traçabilité et évolution d'une pollution métallurgique médiévale de plomb argentifere sur le Mont Lozère, Thèse de Doctorat, Université de Montpellier II, France.

Baron, S., Lavoie, M., Ploquin, A., Carignan, J., Lozère, M. et DE BeAulieu, J. -L., 2005. - Record of Metal Workshops in Peat Deposits : History and Environmental Impact on the Mont-Lozère Massif (France), Environmental Science and Technology, 39, p. 5131-5140.

Baron, S., Carignan, J., Laurent, S. et Ploquin, A., 2006a. - Medieval lead making on Mont-Lozère Massif (CévennesFrance) : tracing ore sources by using $\mathrm{Pb}$ isotopes, Applied Geochemistry, 21, p. 241-252.

Baron, S., Carignan, J. et Ploquin, A., 2006b. - Dispersion of Heavy metals (Metaloids) in Soils from 800-Year-Old Pollution (Mont-Lozère, France), Environmental Science and Technology, 40, p. 5319-5326.

Baron, S., Carignan, J. et Ploquin, A., 2007. - Dispersion de métaux lourds dans l'environnement d'ateliers médiévaux (Mont Lozère, France), L’Actualité Chimique, 305, p. 6-11.

Baron, S., Le-Carlier, C., Carignan, J. et Ploquin, A., 2009. - Archaeological reconstruction of medieval lead production : Implications for ancient metal provenance studies and paleopollution tracing by $\mathrm{Pb}$ isotopes, Applied Geochemistry, 24, p. 2093-2101.

Biringuccio, V., 1540. - De la Pirotechnica, Trad. Smith, C. S. and Gnudi, M. T. 1959.

Budd, P., Pollard, A. M., Scaiffe, B. et Thomas, R. G., 1995. - The possible fractionation of lead isotopes in ancient metallurgical processes, Archaeometry, 37, 1, p. 143-150.
Faure, G., 1986. - Principles of Isotopes Geology, London, John Wiley and Sons ed.

Jouffroy-Bapicot, I., Pulido, M., Baron, S., Galop, D., Monna, F., Lavoie, M., Ploquin, A., Petit, C., De Beaulieu, J.-L. et RicharD, H., 2006. - Environmental impact of early palaeometallurgy : pollen and geochemical analysis, Vegetation History and Archaeobotany, 16, p. 251-258.

Laurent, S., 2005. - Mines et Métallurgie du plomb argentifere sur le Mont-Lozère au Moyen Âge (XI $-X V^{e}$ siècles), Mémoire de Maîtrise, Université de Lyon II, vol. 1 et 2, France.

Monna F., Petit C. Guillaumet, J.-P., Jouffroy-Bapicot I., Blanchot C., Dominik J., Losno R., Richard H., LeVeque J. et Château, C., 2004a. - History and environmental impact of mining activity in Celtic Aeduan territory recorded in a peat bog (Morvan, France), Environmental Science and Technology, 38, p. 665-673.

Monna F., Galop D., Carozza L., Tual M., Beyrie A., Marembert F., Chateau C., Dominik J. et Grousset F. E., 2004b. - Environmental impact of early Basque mining and smelting recorded in a hight ash minerogenic peat deposit, Science of the Total Environment, 327, p. 197-214.

Nriyagu, J. O. et Pacyna, J. M., 1988. - Quantitative assessment of worlwide contamination of air, water and soils by trace metals, Nature, 333, p. 134-139.

Ploquin, A., Allée, Ph., Bailly-Maître, M.-Ch., Baron, S., de Beaulieu, J.-L., Carignan, J., Laurent, S., Lavoie M., Mahé-le Carlier, C., Peytavin, J., Pulido, M., 2003. Medieval lead smelting on the Mont-Lozère, Southern France, International Conference in Archaeometallurgy in Europe, Milan, Italy, 24-26 Septembre 2003, 1, p. 635-644.

Pulido, A. M., 2006. - Conséquences de l'anthropisation sur la dynamique post-glaciaire de la végétation dans le sud du Massif Central, France, Thèse de doctorat, Université Aix-Marseille, France. 
